\title{
Gas-Dynamical Modelling of Circumstellar Matter in $\beta$ Lyr A
}

D. V. Bisikalo and A. A. Boyarchuk

Institute of Astronomy, Russian Academy of Sciences, Pyatnitskaya 48, Moscow 109017, Russia

P. Harmanec ${ }^{1}$ and P. Hadrava ${ }^{2}$

Astronomical Institute, Academy of Sciences of the Czech Republic, CZ-251 65 Ondřejov, Czech Republic

\section{O. A. Kuznetsov}

Keldysh Institute of Applied Mathematics, Russian Acad. of Sciences, Miusskaya sq. 4, Moscow 125047, Russia

\begin{abstract}
Available spectroscopic evidence of various circumstellar gaseous structures in the well-known binary $\beta$ Lyr is confronted with the results of 3-D gas-dynamical simulations of mass transfer between the binary components, obtained for the currently best available basic physical properties of the system. It appears that the gas-dynamical model is capable to explain, at least qualitatively, the formation of all basic observed components of the circumstellar matter, including the jet-like structures, recently found from interferometry and polarimetry of the object.
\end{abstract}

\section{Introduction}

The spectroscopic and eclipsing binary $\beta$ Lyr A (HD 174638) is the brightest member of an optical system of six stars. According to the ephemeris derived by Harmanec \& Scholz (1993), the orbital period of 12.9-d is growing at a rate of $19 \mathrm{~s}$ per year. The presence of the circumstellar matter in the binary is documented by the complicated line spectrum consisting of at least six different systems of spectral lines:

1. The B6-8II spectrum

2. The B-type "shell" absorption spectrum

3. The emission spectrum

4. The satellite spectrum

5. Secondary Si II 2 absorption spectrum

6. The interstellar lines

\footnotetext{
${ }^{1}$ Present Address: Institute of Astronomy, Charles University, V Holešovičkách 2,
} CZ-180 00 Praha 8, Czech Republic

${ }^{2}$ NTNU, Trondheim, Norway 
The binary consists of the following two stars:

(1) the more massive star 1 (probably of an early-B spectral type) which is completely (or almost completely) hidden from view by a thick accretion disk (see the right panel of Fig. 2), and

(2) the less massive B6-8II star 2 which, however, is the brighter of the two in the optical region. This is so because of its large radius and because the light of star 1 is obscured by the circumstellar disk and re-radiated away from the orbital plane.

The primary eclipse of star 2 by star 1 and its disk is about $1^{\mathrm{m}} .0$ deep in the visual; the secondary eclipse is $0^{\mathrm{m}} \cdot 5$ deep.

\section{Jets}

The B-type "shell" spectrum was originally believed to belong to the star 1 and later to originate in an expanding envelope around the binary system (see, e.g., Struve 1941). However, Harmanec (1992) pointed out that there is not enough radiative flux to excite $\mathrm{He} I$ lines in the orbital plane outside the binary.

Using the optical spectro-interferometry, Harmanec et al. (1996) discovered that most of the emission originates in bipolar jet-like structures above the orbital plane and they tentatively suggested that the "shell" lines arise in self-absorption in these jets. The phase-shift of radial velocities of these lines indicates that the jets emanate from an area of probable interaction of the gas stream from star 2 with the disk around star 1.

The jet-like structures were independently found from spectropolarimetry by Hoffman et al. (1998).

\section{KOREL analysis}

The code KOREL for spectral disentangling and relative line photometry (Hadrava 1995,1997$)$ was used to measure radial velocities and to decompose the contributions in the observed spectra connected with the motion of both components (and the telluric lines). To minimize the influence of the complex structure observed in the strong $\mathrm{H}$ and $\mathrm{He}$ lines, the pair of weak Si II 2 lines was chosen for the analysis of 68 Ondrejov reticon spectra. Despite the fact that the assumption of constant shape of the lines (on which the disentangling method is based) is obviously violated in interacting binaries, the spectra were decomposed as shown in the left panel of Fig. 1 . The line profile moving with star 1 shows a double-peaked emission with a central reversal.

The corresponding RV curves are also shown in Fig. 1. The scatter of radial velocities of star 1 results from the variability of the profile and perhaps from the rotation effect and the contribution of satellite lines. The KOREL solution of orbital elements gives $K_{1}=42.1 \mathrm{~km} \mathrm{~s}^{-1}, K_{2}=186.8 \mathrm{~km} \mathrm{~s}^{-1}$, in a good agreement with Harmanec \& Scholz (1993), $M_{1} / M_{2}=4.432$. 

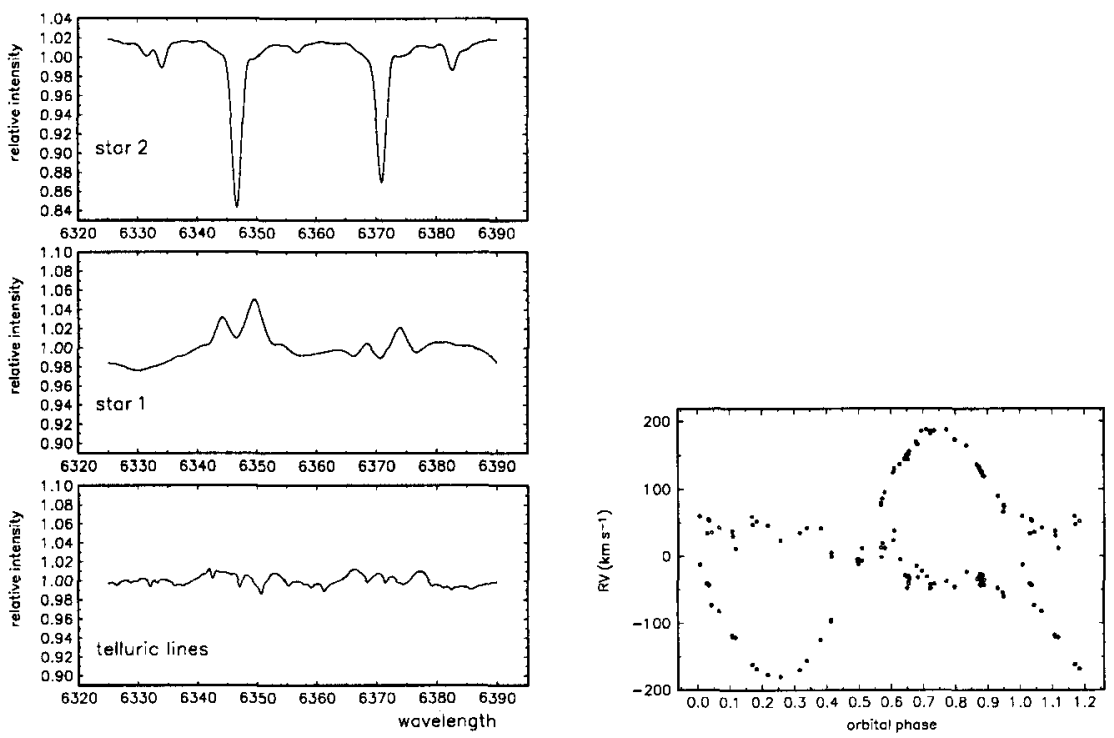

Figure 1. Left: Decomposed line profiles of both binary components of $\beta$ Lyr and of the telluric spectrum in the neighbourhood of Si II 2 lines. Right: The RV curves of both components which resulted from KOREL disentangling orbital solution; open circles correspond to the RV curve of star 1
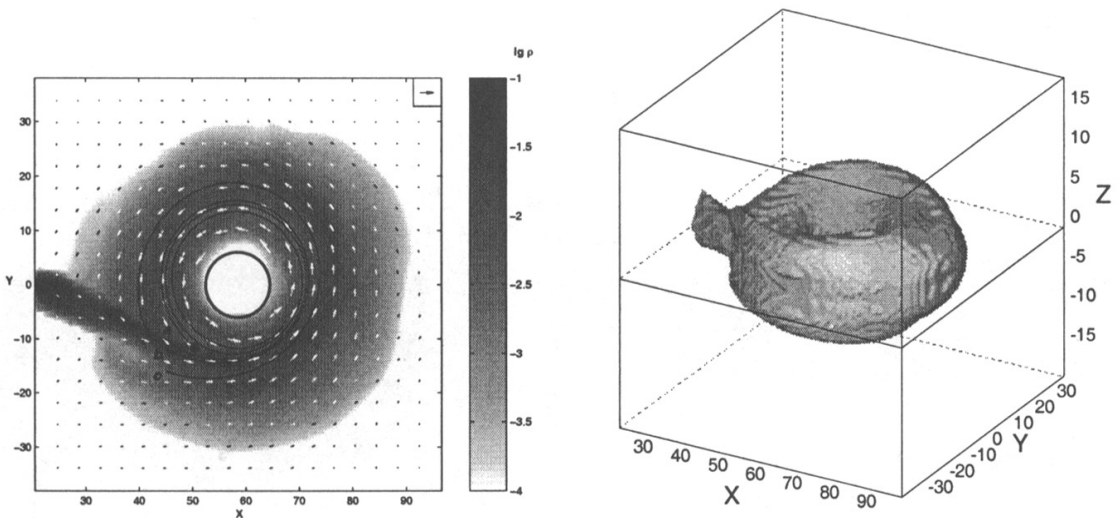

Figure 2. Left: Density distribution and the velocity vectors in the orbital plane of the binary system. A scaling vector in the upper right corner corresponds to velocity of $500 \mathrm{~km} \mathrm{~s}^{-1}$. Right: A bird-eye view of the density iso-surface at the level $\rho=1 \cdot 10^{-3} \rho\left(L_{1}\right)$. All coordinates are scaled in units of $R_{\odot}$. 


\section{Model}

The method for numerical 3-D gas-dynamical modelling described in detail by Bisikalo et al. $(1997,1998)$ has been used to investigate the structure of the circumstellar matter around the star 1 . The Euler equations and the equation of state of the ideal gas with the adiabatic index $\gamma=1.01$ (i.e. nearly isothermal case) are solved in the Cartesian grid $61 \times 61 \times 17$ points.

The following physical elements of the binary were adopted: $M_{1}=13.2 \mathrm{M}_{\odot}, R_{1}=6 \mathrm{R}_{\odot}, M_{2}=2.98 \mathrm{M}_{\odot}, T_{2}=12000 \mathrm{~K}, A=58.5 \mathrm{R}_{\odot}$, star 2 fills its Roche lobe.

Analysis of the gas-dynamical solution shows that the main part of the energy release occurs near the point $X=50.4 \mathrm{R}_{\odot}, Y=-11.55 \mathrm{R}_{\odot}$ labelled by a filled circle in the left panel of Fig. 2. This coincides with the location where the "jets" start, calculated by Harmanec et al. (1996) as the "optical centre" of the blue-shifted absorption lines from the phase shift of their RV curves with respect to the RV curves of the binary components. These authors suggested that the spectral lines in question mainly arise from the self-absorption in one of the jet-like structures which they found from optical spectro-interferometry.

The presence of a normal stellar wind emanating from the polar regions of an early-B star 1 is to be expected which is not included in the present gasdynamical model. This wind must also move mainly along the directions perpendicular to the orbital plane since in all other directions it interacts with the envelope and looses its identity. A self-consistent radiation-hydrodynamic modelling of the circumstellar matter as well as very high-resolution spectroscopic observations of the system are thus needed for further progress of understanding of the system in the future.

Acknowledgments. This work has been supported by the grant A3003805 of GA AVČR and by grants RFBR 99-02-17619 and 99-15-96022 by President of Russian Federation.

\section{References}

Bisikalo, D.V., Boyarchuk, A.A., Kuznetsov, O.A., Chechetkin, V.M. 1997, AZh. 74, 880 (Astron. Report 41, 786)

Bisikalo, D.V., Boyarchuk, A.A., Chechetkin, V.M., Kuznetsov, O.A., Molteni, D. 1998, MNRAS 300, 39

Hadrava, P. 1995, A\&AS 114, 393

Hadrava, P. 1997, A\&AS 122, 581

Harmanec, P. 1992, A\&A 266, 307

Harmanec, P., Scholz G. 1993, A\&A 279, 131

Harmanec, P., Morand, F., Bonneau, D., Jiang, Y., Yang, S., Guinan, E.F., Hall, D.S., Mourard, D., Hadrava, P., Božić H., Sterken, C., Tallon-Bosc, I., Walker, G.A.H., McCook, G.P., Vakili, F., Stee, Ph., Le Contel, J.M. 1996, A\&A 312, 879

Hoffman, J.L., Nordsieck, K.H., Fox, G.K. 1998, AJ 115, 1576

Struve, O. 1941, ApJ 93, 104 